\title{
Identification of Synthetic Organic Pigments (SOPs) Used in Modern Artist's Paints with Secondary Ion Mass Spectrometry with MeV Ions
}

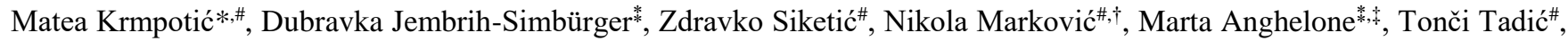
Dora Plavčić»\$, Mason Malloy ${ }^{\S}$, Iva Bogdanović Radović\#

\#Division of Experimental Physics, Laboratory for Ion Beam Interactions, Ruđer Bošković Institute, Bijenička cesta 54, HR10000 Zagreb, Croatia

${ }^{*}$ Institute of Science and Technology in Art, Academy of Fine Arts Vienna, Schillerplatz 3, A-1010 Vienna, Austria

Department of Physics, Faculty of Science, University of Zagreb, Bijenička cesta 32, HR-10000 Zagreb, Croatia

${ }^{8}$ Department of Chemistry, University of Surrey, Guildford, Surrey, GU2 7XH, United Kingdom

*E-mail: Matea.Krmpotic@irb.hr

\section{Present Addresses}

'Department of Radiation Physics, Institute of Clinical Sciences Sahlgrenska Academy, University of Gothenburg, P.O. Box 426 SE405 30 Gothenburg, Sweden

Institute of Conservation, University of Applied Arts Vienna, Salzgries 14, A-1010 Vienna, Austria

§Obrnuta faza d.o.o., Krapinska 36, HR-10000 Zagreb, Croatia

\section{Table of Contents}

Table S-1. List of characteristic ions of the blue and green $\mathrm{Cu}$ phthalocyanine pigments detected with $\mathrm{MeV}$ SIMS (5 $\mathrm{MeV} \mathrm{Si}^{4+}$, positive-ion mode)

Table S-2. List of characteristic ions of the yellow, violet, red and orange pigments detected with MeV-SIMS (5 $\mathrm{MeV} \mathrm{Si}^{4+}$, positive-ion mode)

Table S-3. List of SOPs' characteristic ions detected with MeV-SIMS (5 $\mathrm{MeV} \mathrm{Si}^{4+}$, positive-ion mode) in the selected commercial tube paints

For detailed description of the analyzed pigments please see the Primary Research Article. 
Table S-1. List of characteristic ions of the blue and green $\mathrm{Cu}$ phthalocyanine pigments detected with MeV-SIMS (5 MeV $\mathrm{Si}^{4+}$, positive-ion mode); the most abundant peaks in the spectra are in bold

\begin{tabular}{|c|c|c|c|c|}
\hline C.I. name & Substituents & $m / z(\mathrm{FWHM})$ & Fragment ion & Fragment ion structure \\
\hline \multirow{9}{*}{$\begin{array}{l}\text { PB15:1 } \\
\text { PB15:3 } \\
\text { PB15:6 }\end{array}$} & \multirow{9}{*}{$* 0.5-1 \mathrm{Cl}$} & $\mathbf{5 7 5 , 5 7 6}(3.6-4.7)$ & {$[\mathrm{M}]^{+\bullet},[\mathrm{M}+\mathrm{H}]^{+}$} & copper phthalocyanine \\
\hline & & $609,611^{*}(5.7,5.7)$ & {$\left[\mathrm{C}_{32} \mathrm{H}_{15} \mathrm{ClCuN}_{8}\right]^{+\bullet}$} & copper phthalocyanine \\
\hline & & $191,193(1.0-3.8)$ & {$\left[\mathrm{C}_{8} \mathrm{H}_{4} \mathrm{CuN}_{2}\right]^{+\bullet}$} & copper benzene-1,2-dicarbonitirile \\
\hline & & $165,167(0.6-1.4)$ & {$\left[\mathrm{C}_{7} \mathrm{H}_{4} \mathrm{CuN}\right]^{+}$} & copper benzonitrile \\
\hline & & $152,154(0.4-0.9)$ & ${ }^{* *}\left[\mathrm{C}_{7} \mathrm{H}_{5} \mathrm{Cu}\right]^{+}$ & copper cyclohepta-1,3,5-triene ${ }^{* *}$ \\
\hline & & $128,129(0.4-2.1)$ & {$\left[\mathrm{C}_{8} \mathrm{H}_{4} \mathrm{~N}_{2}\right]^{+\bullet},\left[\mathrm{C}_{8} \mathrm{H}_{5} \mathrm{~N}_{2}\right]^{+}$} & benzene-1,2-dicarbonitirile \\
\hline & & $114,115(0.4-0.9)$ & ${ }^{* *}\left[\mathrm{C}_{8} \mathrm{H}_{4} \mathrm{~N}\right]^{+},\left[\mathrm{C}_{8} \mathrm{H}_{5} \mathrm{~N}\right]^{+\bullet}$ & $\begin{array}{l}\text { 2-phenylethenylideneazanide } \\
\text { (cyanophenylcarbene) }^{* *}\end{array}$ \\
\hline & & $102,103(0.4-1.9)$ & {$\left[\mathrm{C}_{7} \mathrm{H}_{4} \mathrm{~N}\right]^{+},\left[\mathrm{C}_{7} \mathrm{H}_{5} \mathrm{~N}\right]^{+\bullet}$} & benzonitrile \\
\hline & & $63,65(0.3-0.6)$ & $\mathrm{Cu}^{+}$ & \\
\hline \multirow{4}{*}{$\begin{array}{l}\text { PB16 } \\
\text { (metal free) }\end{array}$} & \multirow{4}{*}{-} & $514(4.7)$ & {$[\mathrm{M}]^{+\bullet}$} & phthalocyanine \\
\hline & & $129(1.2)$ & {$\left[\mathrm{C}_{8} \mathrm{H}_{5} \mathrm{~N}_{2}\right]^{+}$} & benzene-1,2-dicarbonitirile \\
\hline & & $114,115(0.7,0.7)$ & ${ }^{* *}\left[\mathrm{C}_{8} \mathrm{H}_{4} \mathrm{~N}\right]^{+},\left[\mathrm{C}_{8} \mathrm{H}_{5} \mathrm{~N}\right]^{+\bullet}$ & $\begin{array}{l}\text { 2-phenylethenylideneazanide } \\
\text { (cyanophenylcarbene) }^{* *}\end{array}$ \\
\hline & & $103(1.1)$ & {$\left[\mathrm{C}_{7} \mathrm{H}_{5} \mathrm{~N}\right]^{+\bullet}$} & benzonitrile \\
\hline \multirow{10}{*}{ PG7 } & \multirow{10}{*}{$\begin{array}{l}\mathrm{Cl} \text { on benzene } \\
\text { rings }\end{array}$} & $1127(12.5)$ & {$[\mathrm{M}]^{+\bullet}$} & copper perchlorophthalocyanine \\
\hline & & $\begin{array}{l}1092-915 \\
(12.5-13.8)\end{array}$ & $\begin{array}{l}\text { a series of molecular ion peaks } \\
\text { corresponding to different } \\
\text { ratios of } \mathrm{Cl} \text { atoms substituted } \\
\text { on benzene rings (from } 16 \text { to } \\
10 \mathrm{Cl} \text { atoms) }\end{array}$ & \\
\hline & & $576(3.1)$ & {$\left[\mathrm{C}_{32} \mathrm{H}_{17} \mathrm{CuN}_{8}\right]^{+}$} & copper phthalocyanine \\
\hline & & $328-331(6.0)$ & {$\left[\mathrm{C}_{8} \mathrm{HCl}_{4} \mathrm{CuN}_{2}\right]^{+}$} & copper tetrachlorobenzene-1,2-dicarbonitrile \\
\hline & & $292-295(5.0)$ & {$\left[\mathrm{C}_{8} \mathrm{Cl}_{3} \mathrm{CuN}_{2}\right]^{+}$} & copper trichlorobenzene-1,2-dicarbonitrile \\
\hline & & $264-270(4.0)$ & {$\left[\mathrm{C}_{8} \mathrm{Cl}_{4} \mathrm{~N}_{2}\right]^{+\bullet}$} & tetrachlorobenzene-1,2-dicarbonitrile \\
\hline & & $214-220(0.6)$ & {$\left[\mathrm{C}_{6} \mathrm{H}_{2} \mathrm{Cl}_{4}\right]^{+\bullet}$} & tetrachlorobenzene \\
\hline & & $155,157(2.9,3.1)$ & ${ }^{* *}\left[\mathrm{C}_{7} \mathrm{H}_{8} \mathrm{Cu}\right]^{+}$ & copper cyclohepta-1,3,5-triene ${ }^{* *}$ \\
\hline & & $115(0.8)$ & ${ }^{* *}\left[\mathrm{C}_{8} \mathrm{H}_{5} \mathrm{~N}\right]^{+\bullet}$ & $\begin{array}{l}\text { 2-phenylethenylideneazanide } \\
\text { (cyanophenylcarbene) }^{* *}\end{array}$ \\
\hline & & $\mathbf{6 3 ,} 65(0.7,0.8)$ & $\mathrm{Cu}^{+}$ & \\
\hline \multirow[t]{3}{*}{ PG36 } & \multirow[t]{3}{*}{$\begin{array}{l}\mathrm{Br}, \mathrm{Cl} \text { on } \\
\text { benzene rings }\end{array}$} & $\begin{array}{l}1839-992 \\
(11.4-22.9)\end{array}$ & $\begin{array}{l}\text { a series of molecular ion peaks } \\
\text { corresponding to different } \\
\text { ratios of } \mathrm{Br} \text { and } \mathrm{Cl} \text { atoms } \\
\text { substituted on benzene rings } \\
\text { (from } 16 \mathrm{Br}, 0 \mathrm{Cl} \text { to } 0 \mathrm{Br}, 12 \mathrm{Cl} \text { ) }\end{array}$ & \\
\hline & & $503-515(7.7)$ & {$\left[\mathrm{C}_{8} \mathrm{Br}_{4} \mathrm{CuN}_{2}\right]^{+}$} & copper tetrabromobenzene-1,2-dicarbonitrile \\
\hline & & $\mathbf{6 3}, \mathbf{6 5}(0.7,0.9)$ & $\mathrm{Cu}^{+}$ & \\
\hline
\end{tabular}

* present only in PB15:1 pigment

** proposed fragment ion; indium ion detection at $\mathrm{m} / \mathrm{z} 115$ may be possible 
Table S-2. List of characteristic ions of the yellow, violet, red and orange pigments detected with $\mathrm{MeV}$-SIMS (5 $\mathrm{MeV} \mathrm{Si}^{4+}$, positive-ion mode); the most abundant peaks in the spectra are in bold

\begin{tabular}{|c|c|c|c|c|}
\hline C.I. name & $\begin{array}{l}\text { Pigment class } \\
\text { / substituents }\end{array}$ & $m / z($ FWHM) & Fragment ion & Fragment ion structure \\
\hline \multirow{7}{*}{ PY3 } & \multirow{7}{*}{$\begin{array}{l}\text { monoazo } \\
\text { R1: } \mathrm{Cl} \\
\text { R2: } \mathrm{NO}_{2} \\
\text { R3: } \mathrm{Cl}\end{array}$} & $\mathbf{3 9 4}, \mathbf{3 9 5}(4.0,4.4)$ & {$[\mathrm{M}]^{+\bullet},[\mathrm{M}+\mathrm{H}]^{+}$} & $\begin{array}{l}\text { 2-[(4-chloro-2-nitrophenyl)diazenyl]- } N \text {-(2- } \\
\text { chlorophenyl)-3-oxobutanamide }\end{array}$ \\
\hline & & $378(2.0)$ & {$[\mathrm{M}-\mathrm{O}]^{+}$} & \\
\hline & & $268,270(1.2,2.1)$ & {$\left[\mathrm{C}_{10} \mathrm{H}_{7} \mathrm{~N}_{3} \mathrm{O}_{4} \mathrm{Cl}\right]^{+}$} & product of the $[\mathrm{M}]^{+}$amide bond cleavage \\
\hline & & $171,173(4.0,2.0)$ & {$\left[\mathrm{C}_{6} \mathrm{H}_{4} \mathrm{~N}_{2} \mathrm{O}_{2} \mathrm{Cl}\right]^{+}$} & p-chloro-o-nitroaniline \\
\hline & & $154,156(1.0,1.0)$ & {$\left[\mathrm{C}_{7} \mathrm{H}_{5} \mathrm{NOCl}\right]^{+}$} & 1-chloro-2-isocyanatobenzene \\
\hline & & $\mathbf{1 2 7}, \mathbf{1 2 9}(0.8,4.0)$ & {$\left[\mathrm{C}_{6} \mathrm{H}_{6} \mathrm{NCl}\right]^{+}$} & o-chloroaniline \\
\hline & & $111,113(0.8,0.7)$ & {$\left[\mathrm{C}_{6} \mathrm{H}_{4} \mathrm{Cl}\right]^{+}$} & chlorobenzene \\
\hline \multirow{5}{*}{ PY74 } & \multirow{5}{*}{$\begin{array}{l}\text { monoazo } \\
\text { R1: } \mathrm{OCH}_{3} \\
\text { R2: } \mathrm{OCH}_{3} \\
\text { R3: } \mathrm{NO}_{2}\end{array}$} & $\mathbf{3 8 6}, \mathbf{3 8 7}(2.7,3.0)$ & {$[\mathrm{M}]^{+\bullet},[\mathrm{M}+\mathrm{H}]^{+}$} & $\begin{array}{l}\text { 2-[(2-methoxy-4-nitrophenyl)diazenyl]- } N \text {-(2- } \\
\text { methoxyphenyl)-3-oxobutanamide }\end{array}$ \\
\hline & & $369(1.3)$ & {$[\mathrm{M}-\mathrm{O}-\mathrm{H}]^{+}$} & \\
\hline & & $168(2.4)$ & {$\left[\mathrm{C}_{7} \mathrm{H}_{8} \mathrm{~N}_{2} \mathrm{O}_{3}\right]^{\bullet}$} & 2-methoxy-4-nitroaniline \\
\hline & & $152(1.3)$ & {$\left[\mathrm{C}_{7} \mathrm{H}_{6} \mathrm{NO}_{3}\right]^{+}$} & 1-methoxy-3-nitrobenzene \\
\hline & & $123(0.7)$ & {$\left[\mathrm{C}_{7} \mathrm{H}_{9} \mathrm{NO}^{+}\right]^{\bullet}$} & o-methoxyaniline \\
\hline \multirow{4}{*}{ PV19 } & \multirow{4}{*}{$\begin{array}{l}\text { quinacridone } \\
\text { R1: H } \\
\text { R2: H }\end{array}$} & $312(3.5)$ & {$[\mathrm{M}]^{+\bullet}$} & $\begin{array}{l}\text { 5,12-dihydroquinolino[2,3-b]acridine-7,14- } \\
\text { dione }\end{array}$ \\
\hline & & $295(5.4)$ & {$[\mathrm{M}-\mathrm{OH}]^{+}$} & \\
\hline & & $284(4.6)$ & {$[\mathrm{M}-\mathrm{CO}]^{+}$} & \\
\hline & & $256(3.9)$ & {$[\mathrm{M}-2 \mathrm{CO}]^{+}$} & \\
\hline \multirow{8}{*}{ PR122 } & \multirow{8}{*}{$\begin{array}{l}\text { quinacridone } \\
\mathrm{R} 1: \mathrm{CH}_{3} \\
\mathrm{R} 2: \mathrm{CH}_{3}\end{array}$} & $341(4.0)$ & {$[\mathrm{M}+\mathrm{H}]^{+}$} & $\begin{array}{l}\text { 2,9-dimethyl-5,12-dihydroquinolino[2,3- } \\
\text { b]acridine-7,14-dione }\end{array}$ \\
\hline & & $325,326(4.1,4.0)$ & {$\left[\mathrm{M}-\mathrm{CH}_{3}\right]^{+},\left[\mathrm{M}-\mathrm{CH}_{3}+\mathrm{H}\right]^{+} \bullet$} & \\
\hline & & $312(5.0)$ & {$\left[\mathrm{C}_{20} \mathrm{H}_{12} \mathrm{~N}_{2} \mathrm{O}_{2}\right]^{+\bullet}$} & $\begin{array}{l}\text { 5,12-dihydroquinolino[2,3-b]acridine-7,14- } \\
\text { dione }\end{array}$ \\
\hline & & $295(3.7)$ & {$\left[\mathrm{C}_{20} \mathrm{H}_{11} \mathrm{~N}_{2} \mathrm{O}\right]^{+}$} & {$\left[\mathrm{C}_{20} \mathrm{H}_{12} \mathrm{~N}_{2} \mathrm{O}_{2}-\mathrm{OH}\right]^{+}$} \\
\hline & & $284(5.0)$ & {$\left[\mathrm{C}_{19} \mathrm{H}_{12} \mathrm{~N}_{2} \mathrm{O}\right]^{+}$} & {$\left[\mathrm{C}_{20} \mathrm{H}_{12} \mathrm{~N}_{2} \mathrm{O}_{2}-\mathrm{CO}\right]^{+}$} \\
\hline & & $269(3.4)$ & ${ }^{*}\left[\mathrm{C}_{19} \mathrm{H}_{11} \mathrm{NO}\right]^{+}$ & ${ }^{*}\left[\mathrm{C}_{20} \mathrm{H}_{12} \mathrm{~N}_{2} \mathrm{O}_{2}-\mathrm{HNCO}\right]^{+}$ \\
\hline & & $256(4.0)$ & {$\left[\mathrm{C}_{18} \mathrm{H}_{12} \mathrm{~N}_{2}\right]^{+\bullet}$} & {$\left[\mathrm{C}_{20} \mathrm{H}_{12} \mathrm{~N}_{2} \mathrm{O}_{2}-2 \mathrm{CO}\right]^{+\bullet}$} \\
\hline & & $680-682(5.4)$ & {$[2 \mathrm{M}]^{+}$} & \\
\hline \multirow{7}{*}{ PR177 } & \multirow{7}{*}{ anthraquinone } & $\mathbf{4 4 3}, \mathbf{4 4 4}(2.6,3.5)$ & {$[\mathrm{M}-\mathrm{H}]^{+},[\mathrm{M}]^{+\bullet}$} & $\begin{array}{l}\text { 1-amino-4-(4-amino-9,10-dioxoanthracen-1- } \\
\text { yl)anthracene-9,10-dione }\end{array}$ \\
\hline & & $415,416(1.7,3.7)$ & {$[\mathrm{M}-\mathrm{H}-\mathrm{CO}]^{+\bullet},[\mathrm{M}-\mathrm{CO}]^{+}$} & \\
\hline & & $399(1.4)$ & {$\left[\mathrm{M}-\mathrm{H}-\mathrm{CO}-\mathrm{NH}_{2}\right]^{+}$} & \\
\hline & & $387(2.6)$ & {$[\mathrm{M}-\mathrm{H}-2 \mathrm{CO}]^{+}$} & \\
\hline & & $371(2.2)$ & {$\left[\mathrm{M}-\mathrm{H}-2 \mathrm{CO}-\mathrm{NH}_{2}\right]^{+}$} & \\
\hline & & $358,360(0.4,0.8)$ & $\begin{array}{l}{ }^{*}\left[\mathrm{M}-2 \mathrm{CO}-2 \mathrm{NH}_{2}+2 \mathrm{H}\right]^{+} \\
{ }^{*}\left[\mathrm{M}-2 \mathrm{CO}-2 \mathrm{NH}_{2}+4 \mathrm{H}\right]^{+}\end{array}$ & \\
\hline & & $331,332(1.3,2.1)$ & & unidentified \\
\hline \multirow{6}{*}{ PR112 } & \multirow{6}{*}{$\begin{array}{l}\text { naphthol AS } \\
\text { R1: } \mathrm{CH}_{3} \\
\mathrm{R} 2: \mathrm{Cl} \\
\mathrm{R} 3: \mathrm{Cl} \\
\mathrm{R} 4: \mathrm{Cl}\end{array}$} & $485,486(4.0,4.2)$ & {$[\mathrm{M}]^{\bullet},[\mathrm{M}+\mathrm{H}]^{+}$} & $\begin{array}{l}\text { 3-hydroxy- } N \text {-(2-methylphenyl)-4-[(2,4,5- } \\
\text { trichlorophenyl)diazenyl]naphthalene-2- } \\
\text { carboxamide }\end{array}$ \\
\hline & & $468(4.3)$ & {$[\mathrm{M}-\mathrm{OH}]^{+}$} & \\
\hline & & $\mathbf{3 7 7 ,} \mathbf{3 7 9}(4.8,4.7)$ & {$\left[\mathrm{C}_{17} \mathrm{H}_{8} \mathrm{Cl}_{3} \mathrm{~N}_{2} \mathrm{O}_{2}\right]^{+}$} & product of the $[\mathrm{M}]^{+}$amide bond cleavage \\
\hline & & $286-289^{44}(2.9)$ & & unidentified \\
\hline & & $127-129(0.8-2.6)$ & {$\left[\mathrm{C}_{10} \mathrm{H}_{7}\right]^{+},\left[\mathrm{C}_{10} \mathrm{H}_{8}\right]^{+} \bullet,\left[\mathrm{C}_{10} \mathrm{H}_{9}\right]^{+}$} & naphthalene \\
\hline & & $106,107(0.5,0.3)$ & {$\left[\mathrm{C}_{7} \mathrm{H}_{8} \mathrm{~N}\right]^{+},\left[\mathrm{C}_{7} \mathrm{H}_{9} \mathrm{~N}\right]^{+\bullet}$} & o-methylaniline \\
\hline
\end{tabular}


Table S-2. Continued

\begin{tabular}{|c|c|c|c|c|}
\hline C.I. name & $\begin{array}{l}\text { Pigment class } \\
\text { / substituents }\end{array}$ & $m / z($ FWHM) & Fragment ion & Fragment ion structure \\
\hline \multirow{7}{*}{ PR170 } & \multirow{7}{*}{$\begin{array}{l}\text { naphthol AS } \\
\text { R1: } \mathrm{OCH}_{2} \mathrm{CH}_{3} \\
\text { R2: } \mathrm{H} \\
\text { R3: } \mathrm{CONH}_{2} \\
\text { R4: } \mathrm{H}\end{array}$} & $454,455(3.7,3.9)$ & {$[\mathrm{M}]^{\bullet},[\mathrm{M}+\mathrm{H}]^{+}$} & $\begin{array}{l}\text { 4-[(4-carbamoylphenyl)diazenyl]- } N-(2- \\
\text { ethoxyphenyl)-3-hydroxynaphthalene-2- } \\
\text { carboxamide }\end{array}$ \\
\hline & & $318(2.9)$ & {$\left[\mathrm{C}_{18} \mathrm{H}_{12} \mathrm{~N}_{3} \mathrm{O}_{3}\right]^{+\bullet}$} & product of the $[\mathrm{M}]^{+}$amide bond cleavage \\
\hline & & $274(1.2)$ & {$\left[\mathrm{C}_{17} \mathrm{H}_{10} \mathrm{~N}_{2} \mathrm{O}_{2}\right]^{+}$} & {$\left[\mathrm{C}_{18} \mathrm{H}_{12} \mathrm{~N}_{3} \mathrm{O}_{3}-\mathrm{CONH}_{2}\right]^{+}$} \\
\hline & & $247(3.1)$ & {$\left[\mathrm{C}_{16} \mathrm{H}_{11} \mathrm{~N}_{2} \mathrm{O}\right]^{+}$} & {$\left[\mathrm{C}_{18} \mathrm{H}_{12} \mathrm{~N}_{3} \mathrm{O}_{3}-\mathrm{CONH}_{2}-\mathrm{CO}+\mathrm{H}\right]^{+}$} \\
\hline & & $184(1.6)$ & {$\left[\mathrm{C}_{11} \mathrm{H}_{6} \mathrm{NO}_{2}\right]^{+}$} & $\begin{array}{l}\text { product of the } m / z 318 \text { fragment azo bond } \\
\text { cleavage }\end{array}$ \\
\hline & & $136(2.0)$ & {$\left[\mathrm{C}_{8} \mathrm{H}_{10} \mathrm{NO}\right]^{+}$} & 2-ethoxyaniline \\
\hline & & $128,129(1.8,2.0)$ & {$\left[\mathrm{C}_{10} \mathrm{H}_{8}\right]^{+\bullet},\left[\mathrm{C}_{10} \mathrm{H}_{9}\right]^{+}$} & naphthalene \\
\hline \multirow{7}{*}{ PR254 } & \multirow{7}{*}{$\begin{array}{l}\text { DPP } \\
\text { R1: Cl } \\
\text { R2: Cl }\end{array}$} & $358(4.3)$ & {$[\mathrm{M}+\mathrm{H}]^{+}$} & $\begin{array}{l}\text { 1,4-bis(4-chlorophenyl)-3-hydroxy- } 2 \mathrm{H} \text { - } \\
\text { pyrrolo[3,4-c]pyrrol-6-one }\end{array}$ \\
\hline & & $330(3.6)$ & {$[\mathrm{M}-\mathrm{CO}+\mathrm{H}]^{+}$} & \\
\hline & & $323(4.2)$ & {$[\mathrm{M}-\mathrm{Cl}+\mathrm{H}]^{+}$} & \\
\hline & & $164,166(2.7,4.7)$ & {$\left[\mathrm{C}_{9} \mathrm{H}_{5} \mathrm{ClO}\right]^{\bullet}$} & 3-(4-chlorophenyl)prop-2-ynal \\
\hline & & $\mathbf{1 3 8}, \mathbf{1 4 0}(1.0,3.4)$ & {$\left[\mathrm{C}_{7} \mathrm{H}_{5} \mathrm{ClN}\right]^{+}$} & 4-chlorobenzonitrile \\
\hline & & $130(1.8)$ & {$\left[\mathrm{C}_{9} \mathrm{H}_{6} \mathrm{O}\right]^{+\bullet}$} & 3-phenylprop-2-ynal \\
\hline & & $111,113,115(0.7)$ & $* *$ & unidentified $^{* *}$ \\
\hline \multirow{6}{*}{ PR255 } & \multirow{6}{*}{$\begin{array}{l}\text { DPP } \\
\text { R1: H } \\
\text { R2: H }\end{array}$} & $289(4.2)$ & {$[\mathrm{M}+\mathrm{H}]^{+}$} & $\begin{array}{l}\text { 3-hydroxy-1,4-diphenyl-2H-pyrrolo[3,4- } \\
\text { c]pyrrol-6-one }\end{array}$ \\
\hline & & $262(3.8)$ & {$[\mathrm{M}-\mathrm{CO}+2 \mathrm{H}]^{+}$} & \\
\hline & & $235(4.5)$ & {$[\mathrm{M}-2 \mathrm{CO}+3 \mathrm{H}]^{+}$} & \\
\hline & & $130(2.9)$ & {$\left[\mathrm{C}_{9} \mathrm{H}_{6} \mathrm{O}\right]^{+\bullet}$} & 3-phenylprop-2-ynal \\
\hline & & $113,115(3.5,2.0)$ & $* *$ & unidentified $^{* *}$ \\
\hline & & $104(2.1)$ & {$\left[\mathrm{C}_{7} \mathrm{H}_{6} \mathrm{~N}\right]^{+}$} & benzonitrile \\
\hline \multirow{6}{*}{ PR264 } & \multirow{6}{*}{$\begin{array}{l}\text { DPP } \\
\text { R1: } \mathrm{C}_{6} \mathrm{H}_{5} \\
\mathrm{R} 2: \mathrm{C}_{6} \mathrm{H}_{5}\end{array}$} & $441(3.5)$ & {$[\mathrm{M}+\mathrm{H}]^{+}$} & $\begin{array}{l}\text { 3-hydroxy-1,4-bis(4-phenylphenyl)-2H- } \\
\text { pyrrolo[3,4-c]pyrrol-6-one }\end{array}$ \\
\hline & & $413,414(1.4,1.7)$ & $\begin{array}{l}{[\mathrm{M}-\mathrm{CO}+\mathrm{H}]^{+\bullet}} \\
{[\mathrm{M}-\mathrm{CO}+2 \mathrm{H}]^{+}}\end{array}$ & \\
\hline & & $384,385(1.0,4.3)$ & {$\left[\mathrm{M}-2 \mathrm{CO}^{+\bullet},[\mathrm{M}-2 \mathrm{CO}+\mathrm{H}]^{+}\right.$} & \\
\hline & & $206(4.0)$ & {$\left[\mathrm{C}_{15} \mathrm{H}_{10} \mathrm{O}\right]^{+\bullet}$} & 3-(4-phenylphenyl)prop-2-ynal \\
\hline & & $180(1.5)$ & {$\left[\mathrm{C}_{13} \mathrm{H}_{10} \mathrm{~N}\right]^{+}$} & 4-phenylbenzonitrile \\
\hline & & $153(2.1)$ & {$\left[\mathrm{C}_{12} \mathrm{H}_{9}\right]^{+}$} & phenylcyclohexatriene (biphenyl) \\
\hline \multirow{11}{*}{ PO73 } & \multirow{11}{*}{$\begin{array}{l}\text { DPP } \\
\text { R1: } \mathrm{C}\left(\mathrm{CH}_{3}\right)_{3} \\
\mathrm{R} 2: \mathrm{C}\left(\mathrm{CH}_{3}\right)_{3}\end{array}$} & 400, $401(5.9,6.0)$ & {$[\mathrm{M}]^{+\bullet},[\mathrm{M}+\mathrm{H}]^{+}$} & $\begin{array}{l}\text { 1,4-bis(4-tert-butylphenyl)-3-hydroxy-2H- } \\
\text { pyrrolo[3,4-c]pyrrol-6-one }\end{array}$ \\
\hline & & $385,387(5.7,5.1)$ & {$\left[\mathrm{M}-\mathrm{CH}_{3}\right]^{+},\left[\mathrm{M}-\mathrm{CH}_{3}+2 \mathrm{H}\right]^{+}$} & \\
\hline & & $372(7.1)$ & {$[\mathrm{M}-\mathrm{CO}]^{+}$} & \\
\hline & & $357,358(2.7,3.0)$ & $\begin{array}{l}{\left[\mathrm{M}-\mathrm{CO}-\mathrm{CH}_{3}\right]^{+}} \\
{\left[\mathrm{M}-\mathrm{CO}-\mathrm{CH}_{3}+\mathrm{H}\right]^{+}}\end{array}$ & \\
\hline & & $346(5.4)$ & {$[\mathrm{M}-2 \mathrm{CO}+2 \mathrm{H}]^{+\bullet}$} & \\
\hline & & $331(3.9)$ & {$\left[\mathrm{M}-2 \mathrm{CO}-\mathrm{CH}_{3}+2 \mathrm{H}\right]^{+}$} & \\
\hline & & $185(2.0)$ & {$\left[\mathrm{C}_{13} \mathrm{H}_{13} \mathrm{O}\right]^{+}$} & 3-(4-tert-butylphenyl)prop-2-ynal \\
\hline & & $171(3.0)$ & {$\left[\mathrm{C}_{12} \mathrm{H}_{11} \mathrm{O}\right]^{+}$} & 3-(4-propan-2-ylphenyl)prop-2-ynal \\
\hline & & $160(1.1)$ & {$\left[\mathrm{C}_{11} \mathrm{H}_{14} \mathrm{~N}\right]^{+}$} & 4-tert-butylbenzonitrile \\
\hline & & $145(2.3)$ & {$\left[\mathrm{C}_{10} \mathrm{H}_{11} \mathrm{~N}\right]^{+\bullet}$} & 4-propan-2-ylbenzonitrile \\
\hline & & $130(3.2)$ & {$\left[\mathrm{C}_{9} \mathrm{H}_{6} \mathrm{O}\right]^{+} \bullet$} & 3-phenylprop-2-ynal \\
\hline
\end{tabular}

\footnotetext{
* proposed fragment ion

** indium ion detection at $\mathrm{m} / \mathrm{z}, 113,115$ may be possible
} 
Table S-3. List of SOPs' characteristic ions detected with $\mathrm{MeV}-\mathrm{SIMS}\left(5 \mathrm{MeV} \mathrm{Si}^{4+}\right.$, positive-ion mode) in the selected commercial tube paints; $m / z$ values of the SOP molecular ions are in bold

\begin{tabular}{|c|c|c|c|}
\hline $\begin{array}{l}\text { Type of the } \\
\text { paint }\end{array}$ & Commercial paint product & C.I. name & $m / z$ SOP identifying fragment ions \\
\hline \multirow{4}{*}{ alkyd } & W\&N Phthalo Blue & PB15 & 152, 154 (low), 192, 193, 194, 576, 609 \\
\hline & W\&N Phthalo Green & PG7 & $576,609,952$ (all low intensities) \\
\hline & W\&N Winsor Lemon & PY3 & 127, 129, 154, 156 (low), 268 (low), 395 \\
\hline & W\&N Permanent Rose & PV19 & $256,284, \mathbf{3 1 3}$ \\
\hline \multirow{11}{*}{ acrylic } & W\&N Phthalo Blue (red shade) & PB15:1 & $191,193, \mathbf{5 7 5}, \mathbf{5 7 6}, \mathbf{6 0 9}, \mathbf{6 1 1}$ \\
\hline & Schmincke Phthalo Blue (green shade) & PB15:3 & 115, 129 (low), 191, 193, 575, 576 \\
\hline & LUKAS Phthalo Blue & PB15:6 & $115,129,191,193, \mathbf{5 7 5}, \mathbf{5 7 6}$ \\
\hline & LUKAS Phthalo Turquoise & PB16 & 103 (low), 115, 129, 514, 515 \\
\hline & W\&N Phthalo Green (blue shade) & PG7 & 575 (low) \\
\hline & W\&N Lemon Yellow & PY3 & 127, 129, 154, 156 (low), 267, 269 (low), 376, 396 (low) \\
\hline & W\&N Permanent Rose & PV19 & 256,284 (low), $\mathbf{3 1 2}, \mathbf{3 1 3}$ \\
\hline & W\&N Naphthol Red Light & PR112 & $106,378, \mathbf{4 8 4}$ \\
\hline & W\&N Quinacridone Violet & PR122 & $326,340,341$ \\
\hline & W\&N Pyrrole Red & PR254 & $115,138,140,164,323, \mathbf{3 5 7}, 358$ \\
\hline & W\&N Pyrrole Red Light & PR255 & $104,115,130, \mathbf{2 8 8}, \mathbf{2 8 9}$ \\
\hline
\end{tabular}

